\title{
LOCAL COMPACTNESS AND SIMPLE EXTENSIONS OF DISCRETE SPACES
}

\author{
H. HERRLICH, V. KANNAN AND M. RAJAGOPALAN ${ }^{1}$
}

ABSTRACT. Hereditarily locally compact spaces are characterized as those locally compact spaces which are simple extensions of discrete spaces.

Introduction. If a simple extension of a discrete space is locally compact, then it is hereditarily so. Surprisingly the converse also holds, i.e. every hereditarily locally compact space is a simple extension of a discrete space. The aim of this note is to prove this fact.

Preliminaries. All spaces in question are supposed to be Hausdorff. A dense embedding $e:(X, \mathcal{T}) \rightarrow(Y, \mathcal{S})$ is called a simple extension of $(X, \mathcal{T})$, provided $e[X]$ is open in $(Y, \delta)$ and the subspace of $(Y, \delta)$, determined by the set $Y \backslash e[X]$, is discrete (see B. Banaschewski [1]). A simple extension $e$ : $(X, \mathcal{T}) \rightarrow(Y, \mathcal{S})$ of $(X, \mathcal{T})$ is called a simple local compactification of $(X, \mathcal{T})$, provided $(Y, \delta)$ is locally compact. A simple local compactification $e$ : $(X, \mathcal{J}) \rightarrow(Y, \mathcal{S})$ is called maximal, provided there does not exist any proper local compactification $c:(Y, \mathcal{S}) \rightarrow(Z, \Re)$ such that $c \circ e:(X, \mathcal{T}) \rightarrow(Z, \Re)$ is a simple extension of $(X, \mathscr{T})$. A space $(Y, \mathcal{S})$ is called a (maximal) simple local compactification of a space $(X, \mathcal{J})$, provided there exists a map $e$ : $X \rightarrow Y$ such that $e:(X, \mathcal{T}) \rightarrow(Y, \mathcal{S})$ is a (maximal) simple local compactification.

For any set $A$ and any set $\mathscr{B}$ of infinite subsets of $A$ such that any two members of $\mathscr{B}$ have finite intersection, we will construct a simple local compactification $e_{(A, \mathscr{B})}:(A, \mathscr{P} A) \rightarrow\left(X_{(A, \mathscr{B})}, \mathcal{T}_{(A, \mathscr{B})}\right)$ as follows: (1) $\mathscr{P} A=$ $\{C \mid C \subset A\}$ is the discrete topology on $A$. (2) $X_{(A, \mathscr{B})}$ is the disjoint union of $A$ and $\mathscr{B}$. (3) $e: A \rightarrow X_{(A, \mathscr{B})}$ is the natural embedding, defined by $e(a)=a$ for each $a \in A$. (4) $\widetilde{T}_{(A, \mathscr{B})}$ is the set of those subsets $D$ of $X_{(A, \mathscr{B})}$, satisfying the condition that $B \in D \cap \mathscr{B}$ implies that $B \backslash D$ is finite. [In B. Banaschewski's suggestive terminology [1], $e_{(A, \mathscr{B})}:\left(A, \mathscr{P}_{A}\right) \rightarrow\left(X_{(A, \mathscr{B})}, \mathcal{T}_{(A, \mathscr{B})}\right)$ is the simple extension of the discrete space $(A, \mathscr{P} A)$, determined by the family $\left\{\mathscr{F}_{B} \mid B \in\right.$ $\Re\}$ of trace-filters $\mathscr{F}_{B}=\{C \subset Q \mid B \backslash C$ finite $\}$. $]$

Received by the editors August 11, 1978 and, in revised form, October 17, 1978.

AMS (MOS) subject classifications (1970). Primary 54D45, 54B05, 54 G99.

Key words and phrases. Simple extension, locally compact.

${ }^{1}$ This author was partially supported by NSF grant MCS 7722201. 


\section{Results.}

THEOREM 1. For any space $Y, \delta$ the following conditions are equivalent.

(1) $Y, \mathcal{S}$ is hereditarily locally compact,

(2) $(Y, \mathcal{S})$ is a simple local compactification of a discrete space,

(3) $(Y, \mathcal{S})$ is homeomorphic to a space $\left(X_{(A, \mathscr{B})}, \mathcal{T}_{(A, \mathscr{B})}\right)$ for suitable $A$ and $\mathscr{B}$.

Proof. (1) $\Rightarrow(2)$. Let $X$ be the set of all isolated points of $(Y, \delta)$, let $\mathcal{T}$ be the discrete topology on $X$, and let $e: X \rightarrow Y$ be the natural embedding, defined by $e(x)=x$ for each $x \in X$. We will show that the embedding $e$ : $(X, \mathcal{T}) \rightarrow(Y, \mathcal{S})$ is a simple local compactification. First, assume $e[X]=X$ were not dense in $(Y, \mathcal{S})$. Then there would exist a nonempty, open subset $A$ of $Y \backslash X$ with compact closure. Hence there would exist a sequence of pairwise disjoint open subsets $A_{n}$ of $A$, a sequence of elements $a_{n} \in A_{n}$, and an adherence point $y$ of $\left\{a_{n} \mid n \in \mathbf{N}\right\}$. Consequently the subspace of $(Y, \delta)$, determined by the set $\{y\} \cup \cup\left\{A_{n} \backslash\left\{a_{n}\right\} \mid n \in \mathbf{N}\right\}$, would not be locally compact at $y$, contradicting condition (1). Hence $e:(X, \mathcal{T}) \rightarrow(Y, \mathcal{S})$ is an open, dense embedding. It remains to show that the subspace $(Z, \mathcal{R})$ of $(Y, \delta)$, determined by the set $Z=Y \backslash X$, is discrete. To see this, let $z$ be an element of $Z$. Since the subspace of $(Y, \delta)$, determined by the set $X \cup\{z\}$, is locally compact there exists a neighbourhood $U$ of $z$ in $(Y, \mathcal{S})$ such that $U \cap(X \cup\{z\})$ is compact. This implies $Z \cap \operatorname{int}_{(Y, \delta)} U=\{z\}$, since otherwise $U \cap(X \cup\{z\})$ would not be closed in $(Y, \mathcal{S})$ and hence could not be compact. Therefore $z$ is isolated in $(Z, \Re)$, hence $(Z, \Re)$ is discrete.

$(2) \Rightarrow(3)$. Let $(X, \mathcal{T})$ be a discrete space and $e:(X, \mathcal{T}) \rightarrow(Y, \mathcal{S})$ be a simple local compactification. For each $y \in Y \backslash e[X]$, the set $e[X] \cup\{y\}$ is a neighbourhood of $y$ in $(Y, \delta)$. Hence there exists a compact neighbourhood $K_{y}$ of $y$ in $(Y, \delta)$ with $K_{y} \subset e[X] \cup\{y\}$. Since $e[X]$ is dense in $(Y, \mathcal{S})$ and $(Y, \delta)$ is a Hausdorff space, each set $K_{y}$ is infinite. Since the subspace of $(Y, \delta)$, determined by $K_{y}$, is compact and $e[X]$ consists of isolated points only, every neighbourhood of $y$ meets every infinite subset of $K_{y}$. By the Hausdorffness of $(Y, \delta)$ this implies that $K_{y} \cap K_{z}$ is finite for any two different elements $y$ and $z$ of $Y \backslash e[X]$. With $A=X$ and $\mathscr{B}=\left\{K_{y} \backslash\{y\} \mid y \in\right.$ $Y \backslash e[X]\}$, the extensions $e_{(A, \mathscr{B})}:(X, \mathcal{T}) \rightarrow\left(X_{(A, \mathscr{B})}, \mathcal{T}_{(A, \mathscr{B})}\right)$ and $e(X, \mathcal{T}) \rightarrow$ $(Y, \delta)$ are obviously equivalent. In particular, the spaces $\left(X_{(A, \mathscr{B})}, \mathcal{T}_{(A, \mathscr{B})}\right)$ and $(Y, \delta)$ are homeomorphic.

(3) $\Rightarrow$ (1). Straightforward.

COROLLARY. Every hereditarily locally compact space is scattered, sequential, and an extension of a discrete space, which is simultaneously simple and strict (cf. [1]).

THEOREM 2. For any space $(Y, \mathcal{S})$ the following conditions are equivalent:

(1) $(Y, \delta)$ is a maximal simple local compactification of a discrete space;

(2) $(Y, \mathcal{S})$ is pseudocompact and hereditarily locally compact;

(3) $(Y, \mathcal{S})$ is homeomorphic to a space $\left(X_{(A, \mathscr{B})}, \mathcal{T}_{(A, \mathscr{B})}\right)$, where $A$ is a set and 
$\mathscr{B}$ is a set of infinite subsets of $A$, which is maximal with respect to the property that any two of its members have finite intersection;

(4) $(Y, \mathcal{S})$ is regular, a simple extension of a discrete space, and every closed set of isolated points in $(Y, \mathcal{S})$ is finite.

Proof. $(1) \Rightarrow(2)$. Let $(X, \mathcal{T})$ be a discrete space and let $e:(X, \mathcal{T}) \rightarrow(Y, \delta)$ be a maximal simple local compactification of $(X, \mathcal{T})$. According to Theorem 1 , the space $(Y, \delta)$ is hereditarily locally compact. If it were not pseudocompact, there would exist a sequence $\left(y_{n}\right)$ in $e[X]$ and a continuous map $f$ from $(Y, \delta)$ into the reals with $\lim _{n \rightarrow \infty} f\left(y_{n}\right)=\infty$. This would, in contradiction to (1), allow the construction of a proper local compactification $c:(Y, \delta) \rightarrow$ $(Z, \mathcal{R})$ of $(Y, \mathcal{S})$ such that $c \circ e:(X, \mathcal{T}) \rightarrow(Z, \mathcal{R})$ is simple. As $Z$ one could choose the disjoint union of $Y$ with a singleton set $\left\{z_{0}\right\}$, as $c: Y \rightarrow Z$ the natural embedding, as topology $R$ the set of all subsets $R$ of $Z$ satisfying the following two conditions:

(a) $R \cap Y \in \mathcal{S}$, and

(b) if $z_{0} \in R$ then $\left\{y_{n} \mid n \in \mathbf{N}\right\} \backslash R$ is finite.

$(2) \Rightarrow(3)$. According to Theorem $1,(Y, \delta)$ is homeomorphic to a space $\left(X_{(A, \mathscr{B}}, \mathcal{T}_{(A, \mathscr{B})}\right)$ for suitable $A$ and $\mathscr{B}$. If $\mathscr{B}$ would not be maximal, there would exist an infinite subset $C$ of $A$, meeting each $B \in \mathscr{B}$ in at most finitely many points. Hence $C$ would determine an infinite, clopen, discrete subspace of $\left(X_{(A, \mathscr{B})}, \mathcal{T}_{(A, \mathscr{B})}\right)$, contradicting the pseudocompactness of the latter.

(3) $\Rightarrow(4)$. Straightforward.

$(4) \Rightarrow(1)$. Let $(X, \mathcal{T})$ be a discrete space and let $e:(X, \mathcal{T}) \rightarrow(Y, \mathcal{S})$ be a simple extension of $(X, \mathcal{T})$. For any $y \in Y$, the set $e[X] \cup\{y\}$ is a neighbourhood of $y$ in $(Y, \delta)$. Hence there exists a closed neighbourhood $U$ of $y$ with $U \subset e[X] \cup\{y\}$. For any neighbourhood $V$ of $y$, the set $U \backslash V$ is a closed set of isolated points in $(Y, \mathcal{S})$, and hence finite. Consequently $U$ is a compact neighbourhood of $y$. Thus $e:(X, \mathcal{T}) \rightarrow(Y, \delta)$ is a simple local compactification of $(X, \mathcal{T})$. To show maximality, let $c:(Y, \mathcal{S}) \rightarrow(Z, \Re)$ be a local compactification of $(Y, \delta)$ such that $c \circ e:(X, \mathcal{T}) \rightarrow(Z, \Re)$ is a simple extension of $(X, \mathcal{T})$. Then $c:(Y, \delta) \rightarrow(Z, \Re)$ must be improper, since otherwise there would exist an element $z \in Z \backslash c[Y]$ and a compact neighbourhood $K$ of $z$ in $(Z, \Re)$ with $K \subset c \circ e[X] \cup\{z\}$. Consequently $c^{-1}[K]$ would be an infinite, closed subset of isolated points in $(Y, \delta)$, contradicting condition (4).

\section{REFERENCES}

1. B. Banaschewski, Extensions of topological spaces, Canad. Math. Bull. 7 (1964), 1-22.

Department of Mathematics, University of Bremen, Bremen, Federal Republic of Germany

Department of Mathematics, University of Hyderabad, Hyderabad, INDIA

Department of Mathematics, Universidad de los Andes, Merida, Venezuela 